\title{
Efficacy of proton pump inhibitor on nighttime reflux symptoms and associated sleep disturbances in patients with gastroesophageal reflux disease: A real-world study in northern China
}

\section{Kaidi Sun}

Tianjin Medical University General Hospital https://orcid.org/0000-0003-3247-4043

\section{Shuang Ma}

Tianjin Medical University General Hospital

Yang-Yang Hui

Tianjin Medical University General Hospital

\section{Bin Wang}

Tianjin Medical University General Hospital

Bo Yang

Tianjin Medical University General Hospital

\section{Lan-Ping Zhu}

Tianjin Medical University General Hospital

\section{Sai-Yu Wang}

Tianjin Medical University General Hospital

Shu Li

Tianjin Medical University General Hospital

\section{Wei Zhao}

Tianjin Medical University General Hospital

\section{Kui Jiang}

Tianjin Medical University General Hospital

Jing-Wen Zhao

Tianjin Medical University General Hospital

Bang-Mao Wang ( $\square$ mwang02@tmu.edu.cn)

Tianjin Medical University General Hospital

Xin Chen ( $\nabla$ xchen03@tmu.edu.cn)

Tianjin Medical University https://orcid.org/0000-0003-3024-9053

\section{Guo-Liang Zhang}

Tianjin First Central Hospital

\section{Zheng-Hua Zhou}

First Teaching Hospital of Tianjin University of Traditional Chinese Medicine 


\section{Yan-Di Liu}

Tianjin People's Hospital

\section{Qing Ye}

Tianjin Third Central Hospital

\section{Quan-Jun Deng}

Tianjin Wujing Hospital

\section{Gai-Fang Liu}

Hebei General Hospital

Jun-Min Wang

The Third Hospital of Hebei Medical University

\section{Li-Li Chang}

Shijiazhuang N0.1 Hospital

\section{Rui-Xing Zhang}

The Forth Hospital of Hebei Medical University

\section{Research}

Keywords: Gastroesophageal reflux disease; GERD; nighttime GERD symptoms; sleep disturbance; Proton pump inhibitors

Posted Date: June 9th, 2020

DOI: https://doi.org/10.21203/rs.3.rs-33808/v1

License: (c) (1) This work is licensed under a Creative Commons Attribution 4.0 International License. Read Full License 


\section{Abstract \\ Background}

Gastroesophageal reflux disease (GERD) is strongly associated with sleep disturbances. Proton pump inhibitors (PPIs) are the main treatment to improve the symptom of GERD. This study was to investigate the efficacy of PPIs on nighttime GERD symptoms and associated sleep disturbances in general Chinese GERD population.

\section{Methods}

Adult patients with mild or more serious nighttime GERD symptoms were included. The efficacy was evaluated by the relief rate of nighttime GERD symptoms and GERD-related sleep disturbances after 4 weeks PPIs treatment. Sleep quality was evaluated using the Pittsburgh Sleep Quality Index Score (PSQI).

\section{Results}

A total of 750 patients were included in the final analysis. Overall, $51.3 \%$ of the patients had achieved a relief of nighttime GERD symptoms after 4 weeks of PPIs treatment. By week 4, the percentages of patients with completely resolved nighttime GERD symptoms and GERD-related sleep disturbances were $32.5 \%$ and $51.5 \%$, respectively. PSQI score was significantly improved from $6.82 \pm 0.12$ at baseline to $4.56 \pm 0.09$ at 4 weeks $(P \otimes 0.001)$.

\section{Conclusion}

PPIs treatment can provide an effective relief of nighttime GERD symptoms and associated sleep disturbances, improving sleep quality of general GERD patients in northern China.

\section{Trial registration:}

Trial registration: CHiCTR, ChiCTR1800017652. Registered 08 August 2018, http://chictr.org.cn/index.aspx

\section{Introduction}

Nighttime reflux symptoms of heartburn or acid regurgitation are common among patients with gastroesophageal reflux disease (GERD), affecting about $45-88.9 \%$ of this kind of patients [1-5]. While nighttime reflux episodes are less frequently than daytime reflux episodes, they tend to be associated with more severe reflux-related injuries and bothersome $[2,4,6,7]$. 
Considerable evidences have confirmed the strong association between GERD and sleep disturbances, such as difficultly falling asleep, arousal during sleep and poor sleep quality, which impair the healthrelated quality of life (HRQOL) of patients [2,3,8-12]. In a nationwide telephone survey of 1,000 US patients with GERD, $79 \%$ of respondents experienced nighttime heartburn. In this population, $75 \%$ complained of symptoms that affected their sleep, and $40 \%$ believed that nighttime reflux events impaired their capability of functioning in the next day [3]. In addition, a patient-reported survey of 11,685 respondents with GERD revealed that $88.9 \%$ of the respondents had nighttime reflux symptoms and $68.3 \%$ experienced sleep difficulties. Meanwhile, sleep difficulties were associated with a $5.5 \%$ increase in overall work impairment and a $10.9 \%$ increase in activity impairment [5]. The potential mechanism may be psychoneuroimmunological effect of sleep disturbance [13]. Pool quality of sleep has a great influence on the development of inflammation, cardiovascular disease, carcinogenesis and depression.

Recent studies demonstrate that GERD and GERD-related sleep disturbances were bi-directionally associated. Nighttime reflux symptoms can lead to sleep disturbances, and vice versa sleep disturbances can prolong acid contact time and increase sensory perception $[14,15]$. A survey conducted in China suggested that increased nighttime reflux events might play an important role in inducing sleep disturbances in patients with GERD, and patients with GERD along with sleep disturbances were characterized by more serious nighttime acid reflux and erosive esophagitis [16]. Furthermore, nighttime GRED is related to an increased risk of esophagitis and other respiratory complications [17]. Therefore, it is essential to manage nighttime GERD symptoms and GERD-related sleep disturbances effectively for the improvement of HRQOL as well as the prognosis of patients with GERD.

Treatment involving proton pump inhibitors (PPIs) is the first therapeutic strategy for patients with GERD to relieve GERD symptoms and heal the damaged esophageal mucosa [18]. Several clinical trials have demonstrated that PPIs treatment can reduce nighttime GERD symptoms and improve subjective sleep parameters obviously [9, 19-22]. However, the response of nighttime GERD symptoms and GERD-related

sleep disturbances to PPIs treatment is not well established in China. Therefore, this real-world study was conducted to elucidate the efficacy of PPIs on nighttime GERD symptoms and associated sleep disturbances in general Chinese GERD population.

\section{Materials And Methods 2.1 Design and Setting}

This was a multi-center prospective observational study. The patients from 10 tier-3 hospitals in the north of China from July 2017 to March 2018 were recruited. The study was approved by the ethics committee of Tianjin Medical University General Hospital (approval no. IRB2017-113-01) and conducted in accordance with the ethical principles based on the Declaration of Helsinki and Good Clinical Practice. Written informed consent was obtained from all participants before study initiation.

\subsection{Patients}


We included patients aged from 18 to 80 , who were diagnosed with GERD for the first time or had history of reflux esophagitis but without any acid suppressive agents taken in the previous 4 weeks. The enrolled patients were required to have experienced with typical heartburn or acid regurgitation of at least mild severity. Patients should also have above symptoms for more than one night per week within the previous 3 months. Exclusion criteria were the presence of any conditions other than GERD-related reflux symptoms or sleep disturbances, active peptic ulcer, history of gastroesophageal surgery, suspected or confirmed malignancy, comorbidity of chronic liver diseases and chronic kidney diseases, severe uncontrolled systemic disorders, such as arrhythmia, chronic obstructive pulmonary disease, asthma as well as heart failure, working night shifts, history of drug or alcohol abuse, mental or psychiatric disorder, women who were pregnant or lactating.

\subsection{Assessment}

A non-interventional questionnaire survey was performed in all included patients. The PPIs treatment was prescribed by the physicians before patients were enrolled in the study.

Patients were required to document a diary card to record their daily symptoms and fill out a selfadministered questionnaire after that at each visit. The diary card and questionnaire comprise the questions regarding nighttime GERD symptoms, GERD-related sleep disturbances, the global Pittsburgh Sleep Quality Index (PSQI) questionnaire, and PPIs treatment regimen. Data were collected on baseline, 2 weeks \pm 3 days and 4 weeks \pm 3 days after the initiation of PPIs therapy. All questionnaires were completed with the guidance of attending investigators.

\subsection{Symptom and related sleep disturbance}

Nighttime GERD symptoms were defined as typical heartburn or acid regurgitation when patients laid down to sleep at night, being awakened at night by symptoms, having symptoms on awakening in the morning, and being awakened at night by coughing or choking because of fluid or an acid or bitter taste or food in the throat [14]. On a dairy card, patients were required to answer the following questions related with nighttime GERD symptoms or GERD-related sleep disturbances they had experienced last night, which included "Did you experience nighttime GERD symptoms? (yes or no); Did you have trouble falling asleep or unwanted awakenings caused by nocturnal heartburn or acid regurgitation? (yes or no); Which of the following symptoms caused your trouble sleeping? (heartburn, acid regurgitation)". At each evaluation visit, questionnaires were also required to be completed based on the record of dairy card over the previous 7 day, which included "How many days had you experienced nighttime GERD symptoms? (Please write down the cumulative amount of days); How many days had you experienced sleep trouble due to GERD symptoms? (Please write down the cumulative amount of days)". The patients should also self-assess the severity of nighttime GERD symptoms on a point scale ranging from 0 to 4: none (no symptom); mild (symptoms occasional and easily tolerated); moderate (discomforting symptoms causing interference with normal activities); severe (frequently symptoms cause significant restrictions on daily life), and very sever (sever and persistent life limitations due to symptoms). 
Complete resolution of nighttime GERD symptoms was defined as had never experienced nighttime GERD symptoms in the previous 7 days. Relief was defined as a more than $50 \%$ of reduction in weekly symptomatic days compared with last evaluation visit. Symptom improvement was defined as any decrease compared with last evaluation visit but no more than $50 \%$. Similarly, the improvement of GERDrelated sleep disturbances was defined in the same manner. The efficacy was evaluated by the proportion of patients with relief of nighttime GERD symptoms after 4 weeks of PPIs treatment. A responder was defined as someone who achieved complete or relief status.

\subsection{Sleep quality}

The Pittsburgh Sleep Quality Index Score (PSQI) was used to evaluate subjective sleep quality of subjects [23]. The PSQI is a standardized rating scale, which is composed of seven component scores, including subjective sleep quality, sleep latency, sleep duration, habitual sleep efficiency, sleep disturbances, use of sleeping medication and daytime dysfunction. Each dimension is rated from 0 to 3 , and sum of the seven dimensions yields one global PSQI score. A global PSQI score $₫ 5$ indicates poor sleep.

\subsection{Adverse events}

Adverse events spontaneously reported by the patients were recorded throughout the survey.

\subsection{Statistical analysis}

Analyses were primarily descriptive. Continuous variables with normal distributions were reported as the mean \pm standard deviation (SD). Categorical variables were reported as the percentage. The $c 2$ test was used for the categorical variable. Descriptive comparisons were performed using Wilcoxon's rank-sum tests for continuous variable with non-normally distributed data. Friedman test was used to compare the means of repeated continuous variables. All statistical analyses were performed using SPSS version 25.0 (IBM Corp, Armonk, NY, USA). Statistical significance was defined as two-sided $P<0.05$.

\section{Results}

\subsection{Participants}

Of 1000 patients enrolled in the screening stage, 750 were included in the efficacy evaluations. Flow chart of this study was shown in Fig. 1. The main reasons for the exclusion included early withdraw from the study, major protocol deviation, voluntary discontinuation of PPIs treatment without any post-baseline symptoms, lost of follow-up etc. There were totally 397 male and 353 female with an average age of $51.21 \pm 0.51$ years old. The nighttime heartburn severity score was $1.89 \pm 0.78$, while the PSQI score was $6.82 \pm 0.12$ at baseline. The demographics and baseline characteristics of the 750 patients in the analysis population were presented in Table 1.

\subsection{PPIs treatment}


In the study, all the patients had taken at least one type of PPIs. Among the 750 patients, $70.13 \%$ (526/750) taken PPIs once daily with the standardized dosage recommended by the label information. PPIs treatment strategy was changed during the observation period in $9.1 \%(68 / 750)$ of all patients, of those, $3.20 \%$ (24/750) switched to another type of PPIs, while $5.87 \%(44 / 750)$ of the patients increased the PPIs dose. The most commonly prescribed PPIs was rabeprazole $(69.1 \%, 518 / 750)$, with a mean daily dose of $13.61 \pm 6.63 \mathrm{mg}$. Other PPIs received by patients including esomeprazole $(8.5 \%, 64 / 750)$, lansoprazole $(11.3 \%, 85 / 750)$, pantoprazole $(4.0 \%, 30 / 750)$ and omeprazole $(7.1 \%, 53 / 750)$, with mean daily doses of $26.65 \pm 12.79 \mathrm{mg}, 39.19 \pm 16.01 \mathrm{mg}, 62.67 \pm 41.50 \mathrm{mg}$ and $29.06 \pm 10.05 \mathrm{mg}$, respectively.

\subsection{Effect of PPIs on nighttime GERD symptoms and related sleep disturbances}

Overall, $51.3 \%$ (385/740) patients were considered to be responders of nighttime GERD symptoms after 4 weeks of PPIs treatment. Among them, 32.5\% (244/750) of the patients had achieved complete resolution. The remaining $48.7 \%$ (365/750) of patients showed no response to current treatment. For GERD-related sleep disturbances, 78.0\% (585/750) patients reported sleep disturbances due to nighttime reflux symptoms at baseline. After 4 weeks PPIs treatment, the respondent rate was up to $70.1 \%$ (410/585), while the percentage of patients free from GERD-related sleep disturbances accounted for $53.0 \%(310 / 585)$ of all the patients. Besides, a significantly higher respondent rate of both nighttime GERD symptoms and GERD-related sleep disturbances were achieved at week-4 assessment compared with that at week-2 (34.7\%, 260/750 and 47.0\%, 275/585, respectively) (both $P<0.001$; Fig. 2).

PPIs therapy could significantly decrease the severity of nighttime GERD symptoms $(P<0.001)$. The nighttime heartburn severity score at baseline, week-2 and week- 4 was $1.89 \pm 0.78,1.14 \pm 0.67$ and $0.74 \pm$ 0.67 , respectively. For nighttime acid regurgitation, the mean scores were $1.88 \pm 0.78$ at baseline, $1.07 \pm$ 0.93 at 2 weeks and $0.62 \pm 0.71$ at 4 weeks. Figure 3 showed the change of severity in nighttime symptoms.

\subsection{Effect on sleep quality}

$56.9 \%(427 / 750)$ of patients had poor sleep quality (global PSQI score $>5$ ) at baseline. After PPIs treatment, patients' PSQI score was significantly decreased from $6.82 \pm 0.12$ at baseline to $5.36 \pm 0.09$ at 2 weeks and $4.56 \pm 0.09$ at 4 weeks (both $P<0.001$, Fig. 4A). After 2 weeks PPI treatment, the proportion of patients with a total PSQI score $>5$ decreased to $40.7 \%(305 / 750)(P<0.001)$ and further decreased to $32.7 \%(245 / 750)(P<0.001)$ at 4 weeks (Fig. 4B).

\subsection{Comparison of rabeprazole and non-rabeprazole treatment}

According to the type of PPIs, patients were further divided into rabeprazole group and non-rabeprazole group. At 4 weeks, rabeprazole group had a higher proportion of responders of nighttime GERD symptoms $(57.5 \%$ in rabeprazole vs. $37.5 \%$ in non-rabeprazole, $P<0.001)$. Respondent rate of GERDrelated sleep disturbances was also significantly higher in rabeprazole group $(74.6 \%$ in rabeprazole vs. 
$60.3 \%$ in non-rabeprazole, $P<0.001)$. Similarly, rabeprazole group had a significantly less proportion of patients with poor sleep quality after 4 weeks treatment $(69.9 \%$ in rabeprazole vs. $83.2 \%$ in nonrabeprazole, $P<0.001$ ) (Fig. 5).

\subsection{Adverse events}

In this study, no active safety data were found throughout the survey.

\section{Discussion}

According to a nationwide survey conducted in China, nighttime GERD symptom is common in Chinese GERD patients, accounting for $56.1 \%$ [24]. Among the patients suffering from nighttime GERD symptoms, $80.1 \%$ have sleep disturbances, which is significantly higher than those without nighttime GERD symptoms [24]. Such a high prevalence is comparable to that in western countries. However, the incidence of depression is also increasing with aggravation of sleep disturbances. Although, GERD had been considered as a somatopsychic illness, more and more evidences showed there was a relationship between GERD and sleep-wake cycle and mental disorders $[25,26]$. Thus, sleep management is recommended for patients with GERD to improve the quality of life and avoid the development of psychological problems [27]. This is the first study that provides real-word data to evaluate the efficacy of PPIs on nighttime GERD symptoms and GERD-related sleep disturbances in Chinese patients. With increased understanding of the interrelationship between sleep disturbances and GERD, it may be helpful to provided better therapeutic strategy.

In the present study, 78.0\% patients experienced GERD-related sleep disturbances and $56.9 \%$ patients had poor sleep quality at baseline, which were similar with the results of Zhou et al [24]. Overall, 51.3\% patients in this study had achieved a relief of nighttime GERD symptoms after 4 weeks of PPIs treatment, which was significantly higher than that at 2 weeks (34.7\%). Meanwhile, similar responses to PPIs therapy were also observed in global PSQI score, relief rate of GERD-related sleep disturbances, and complete resolution rate of nighttime GERD symptoms as well as GERD-related sleep disturbances.

The results regarding the efficacy of PPIs therapy on nighttime GERD symptoms and subjective GERDrelated sleep dysfunction in our study were consistent with previous studies. A placebo-controlled study of 864 patients with frequent nighttime heartburn reported that lansoprazole $15 \mathrm{mg}$ or $30 \mathrm{mg}$ once daily were highly effective in reducing nighttime heartburn [28]. However, they didn't evaluate the effect of lansoprazole on sleep. Another multicenter, randomized, double-blind, placebo-controlled trial comparing the efficacy of esomeprazole and placebo in US demonstrated that $53.1 \%$ patients with GERD-related sleep disturbances and moderate-to-severe nighttime heartburn achieved relief of nighttime heartburn, suggesting that esomeprazole $40 \mathrm{mg}$ or $20 \mathrm{mg}$ once daily reduced nighttime heartburn, GERD-related sleep disturbances, and improved sleep quality as well as work productivity in regular activities compared with placebo [20]. Moreover, a multicenter study of 134 GERD patients in Japan found that rabeprazole $10 \mathrm{mg}$ for 8 weeks significantly decreased both FSSG (Frequency Scale for Symptoms of GERD) and PSQI score [9]. Another study in Japan showed that twice daily treatment with rabeprazole for 4 to 8 
weeks significantly reduced the frequency of nighttime reflux symptoms, reflux-related sleep disturbances, daytime sleepiness and improved poor sleep quality in GERD patients who were refractory to once daily standard PPI treatment [29]. The findings from current study suggest that PPIs treatment is effectively in improving nighttime GERD symptoms and GERD-related sleep disturbances over a 4-week period in real-world clinical practice in northern China. Moreover, we found that rabeprazole might be more effective in preventing nighttime GERD symptoms, GERD-related sleep disturbances and improving sleep quality, which was also consistent with previous studies. Compared with other PPIs, higher proportion of patients was treated with rabeprazole and received better relief of night-time symptoms [30, 31]. It may be due to that the intragastric $\mathrm{pH}$ was maintained longer and higher by rabeprazole during the night, thus, central the nervous system received less sensory stimuli from the intraesophageal lumen that may subconsciously interrupt sleep [32]. Rabeprazole may had a faster onset of action than other kinds of PPIs. However, follow-up period in this study was relative short, therefore, we could not observe the long-term effectiveness of different types of PPIs. Besides, it is unknown that whether the patients take the medicine strictly according to doctor's advice. It's also possible that patients may take medicine sometimes during the day and sometimes at night. Patients may take PPIs on the day-time or for several days in the evening. Longer-term clinical trials in patients with GERD would be needed to test those expectations. Therefore, longer term clinical trials of GERD are still needed to answer these questions.

There were several strengths of this study. First, in this large population based real-world study, we firstly evaluated the efficacy of PPIs treatment in patients with nighttime GERD symptoms and GERD-related sleep disturbances in China. These results will help clinicians to effectively manage patients with nighttime GERD symptoms. Second, our results can be presumed to extend to general GERD patients in northern China. Heartburn and acid regurgitation are both typical reflux symptoms according to the Montreal definition of GERD [33]. In addition, acid regurgitation was reported to more frequently occur at night and more severe than heartburn [34,35]. Previous studies exploring the effect of PPIs on nighttime GERD symptoms focused merely on nighttime heartburn [20,22, 28]. In the current study, we enrolled patients with nighttime GERD symptoms with at least mild severity and a frequency of more than one day per week, which represented the majority of GERD patients. Nighttime GERD symptoms included typical heartburn and acid regurgitation. Besides, compared with randomized controlled trials, we included patients with more comorbidities, more concomitant medications and other conditions that were usually excluded by RCTs. Therefore, data in current study can reflect the majority of GERD patients in northern China.

Several limitations need to be considered while interpreting the results in this study. First, data were collected based on patients' self-reported questionnaires, it might be influenced due to recall bias or patient's knowledge of the disease. The available data did not allow consideration of psychological factors and mental disorders that may affect sleep dysfunction. Second, findings in this study mainly represent the situation of target population in northern China, further nationwide researches might be necessary to assess the efficacy of PPIs therapy on the whole target population in China. Third, ambulatory impedance-pH monitoring and polysomnography are not routinely performed in clinical practice. Thus, no objective assessments of nighttime reflux and sleep quality were examined to confirm 
the current results. Hence, additional research is needed to evaluate the objective efficacy in China. In addition, efficacy evaluated in our study was the relief rate of nighttime GERD symptoms after 4 weeks of PPIs treatment. Therefore, not all the patients showing no response to current treatment should be considered as real non-responders. It is possible that some of those patients may experience symptoms improvement in a longer duration of therapy. Thus, further efficacy assessment of long-term PPIs treatment is required. Finally, we were failed to evaluate the confounding factors that might interfere with the current data in real-world setting. The causes of PPIs therapy no-response remains unclear in Chinese GERD patients and investigations concerning this issue are thus warranted.

\section{Conclusions}

In summary, nighttime reflux symptoms are common among Chinese patients with GERD, and most patients have experienced GERD-related sleep disturbances. PPIs treatment can provide an effective relief of nighttime GERD symptoms and associated sleep disturbances, improving sleep quality of general GERD patients in northern China.

\section{List Of Abbreviation}

Gastroesophageal reflux disease (GERD); Proton pump inhibitors (PPIs); Pittsburgh Sleep Quality Index Score (PSQI); Health-related quality of life (HRQOL); The mean \pm standard deviation (SD).

\section{Declarations}

Ethics approval and consent to participate The study was approved by the ethics committee of Tianjin Medical University General Hospital (approval no. IRB2017-113-01) and conducted in accordance with the ethical principles based on the Declaration of Helsinki and Good Clinical Practice. Written informed consent was obtained from all participants before study initiation.

Consent for publication Not applicable

Availability of data and materials The datasets used and/or analysed during the current study are available from the corresponding author on reasonable request.

Founding The study was funded by China International Exchange and Promotive Association for Medical and Health Care [No. ZG-2017-12151019] and supported by Science and Technology Program of Tianjin [19PTZWHZ00090], Natural Science Foundation of Tianjin [18JCZDJC45200], Natural Science Foundation of Tianjin [18JCQNJC80700] and Tianjin Research Innovation Project for Postgraduate Students [2019YJSB108].

Competing interests The authors have no competing of interest to disclose.

Acknowledgments We would like to thank investigators participated in this survey. 
Founding The study was funded by China International Exchange and Promotive Association for Medical and Health Care [No. ZG-2017-12151019] and supported by Science and Technology Program of Tianjin [19PTZWHZ00090], Natural Science Foundation of Tianjin [18JCZDJC45200], Natural Science Foundation of Tianjin [18JCQNJC80700] and Tianjin Research Innovation Project for Postgraduate Students [2019YJSB108].

Authors' contributions KDS, SM and BW participated in the design of this study. YYH and BY performed the statistical analysis. KS and SM drafted the manuscript. BMW, GLZ, ZHZ, YDL, QY, QJD, GFL, JMW, LLC and RXZ provided data. LPZ, SYW, SL, WZ and KJ collected data. JWZ, SM and XC carried out literature search. BMW and $X C$ carried out manuscript editing. KDS, SM, YYH and BW equally contributed to this work. All authors have read and approved the content of the manuscript.

\section{References}

1. Kindt S, Imschoot J, Tack J. Prevalence of and impact of pantoprazole on nocturnal heartburn and associated sleep complaints in patients with erosive esophagitis. Dis Esophagus. 2011;24:531-7.

2. Farup C, Kleinman L, Sloan S, Ganoczy D, Chee E, Lee C, Revicki D. The impact of nocturnal symptoms associated with gastroesophageal reflux disease on health-related quality of life. Arch Intern Med. 2001;161:45-52.

3. Shaker R, Castell DO, Schoenfeld PS, Spechler SJ. Nighttime heartburn is an under-appreciated clinical problem that impacts sleep and daytime function: the results of a Gallup survey conducted on behalf of the American Gastroenterological Association. Am J Gastroenterol. 2003;98:1487-93.

4. Dean BB, Aguilar D, Johnson LF, Fass R, Orr WC, McGuigan JE, Calimlim B, Yan N, Morgenstern D, Dubois RW. The relationship between the prevalence of nighttime gastroesophageal reflux disease and disease severity. Dig Dis Sci. 2010;55:952-9.

5. Mody R, Bolge SC, Kannan H, Fass R. Effects of gastroesophageal reflux disease on sleep and outcomes. Clin Gastroenterol Hepatol. 2009;7:953-9.

6. Fass R. The relationship between gastroesophageal reflux disease and sleep. Curr Gastroenterol Rep. 2009;11:202-8.

7. Chen JH, Wen SH, Hsu CS, Yi CH, Liu TT, Chen CL, Wang CC. Clinical characteristics and psychosocial impact of different reflux time in gastroesophageal reflux disease patients. $\mathrm{J}$ Formos Med Assoc. 2017;116:123-8.

8. Kusano M, Kouzu T, Kawano T, Ohara S. Nationwide epidemiological study on gastroesophageal reflux disease and sleep disorders in the Japanese population. J Gastroenterol. 2008;43:833-41.

9. Fujiwara Y, Kohata Y, Kaji M, Nebiki H, Yamasaki T, Sasaki E, Hayakawa T, Machida H, Tanigawa T, Watanabe K, Watanabe T, Tominaga K, Arakawa T. Sleep dysfunction in Japanese patients with gastroesophageal reflux disease: prevalence, risk factors, and efficacy of rabeprazole. Digestion. 2010;81:135-41. 
10. Jung HK, Choung RS, Talley NJ. Gastroesophageal reflux disease and sleep disorders: evidence for a causal link and therapeutic implications. J Neurogastroenterol Motil. 2010;16:22-9.

11. Okuyama M, Takaishi O, Nakahara K, Iwakura N, Hasegawa T, Oyama M, Inoue A, Ishizu H, Satoh H, Fujiwara Y. Associations among gastroesophageal reflux disease, psychological stress, and sleep disturbances in Japanese adults. Scand J Gastroenterol. 2017;52:44-9.

12. Hyun MK, Baek Y, Lee S. Association between digestive symptoms and sleep disturbance: a crosssectional community-based study. BMC Gastroenterol. 2019;19:34.

13. Irwin MR. Why sleep is important for health: a psychoneuroimmunology perspective. Annu Rev Psychol. 2015;66:143-72.

14. Fujiwara Y, Arakawa T, Fass R. Gastroesophageal reflux disease and sleep disturbances. J Gastroenterol. 2012;47:760-9.

15. Jansson $C$, Nordenstedt $H$, Wallander MA, Johansson S, Johnsen R, Hveem K, Lagergren J. A population-based study showing an association between gastroesophageal reflux disease and sleep problems. Clin Gastroenterol Hepatol. 2009;7:960-5.

16. Hung JS, Lei WY, Yi CH, Liu TT, Chen CL. Association Between Nocturnal Acid Reflux and Sleep Disturbance in Patients With Gastroesophageal Reflux Disease. Am J Med Sci. 2016;352:141-5.

17. Orr WC. Review article: sleep-related gastro-oesophageal reflux as a distinct clinical entity. Aliment Pharmacol Ther. 2010;31:47-56.

18. Chiba N, De Gara CJ, Wilkinson JM, Hunt RH. Speed of healing and symptom relief in grade II to IV gastroesophageal reflux disease: a meta-analysis. Gastroenterology. 1997;112:1798-810.

19. Dimarino AJ Jr, Banwait KS, Eschinger E, Greenberg A, Dimarino M, Doghramji K, Cohen S. The effect of gastro-oesophageal reflux and omeprazole on key sleep parameters. Aliment Pharmacol Ther. 2005;22:325-9.

20. Johnson DA, Orr WC, Crawley JA, Traxler B, McCullough J, Brown KA, Roth T. Effect of esomeprazole on nighttime heartburn and sleep quality in patients with GERD: a randomized, placebo-controlled trial. Am J Gastroenterol. 2005;100:1914-22.

21. Orr WC, Goodrich S, Robert J. The effect of acid suppression on sleep patterns and sleep-related gastro-oesophageal reflux. Aliment Pharmacol Ther. 2005;21:103-8.

22. Johnson D, Crawley JA, Hwang C, Brown K. Clinical trial: esomeprazole for moderate-to-severe nighttime heartburn and gastro-oesophageal reflux disease-related sleep disturbances. Aliment Pharmacol Ther. 2010;32:182-90.

23. Buysse DJ, Reynolds CF 3rd, Monk TH, Berman SR, Kupfer DJ. The Pittsburgh Sleep Quality Index: a new instrument for psychiatric practice and research. Psychiatry Res. 1989;28:193-213.

24. Zou DW, Xu GM. Nocturnal gastric acid reflux and sleep disturbances in patients with gastroesophageal reflux disease. Chin J Dig. 2007;27:828-31.

25. Logan RW, McClung CA. Rhythms of life: circadian disruption and brain disorders across the lifespan. Nat Rev Neurosci. 2019;20:49-65. 
26. Baglioni C, Nanovska S, Regen W, Spiegelhalder K, Feige B, Nissen C, Reynolds CF, Riemann D. Sleep and mental disorders: A meta-analysis of polysomnographic research. Psychol Bull. 2016;142:96990.

27. Gyawali CP, Fass R. Management of Gastroesophageal Reflux Disease. Gastroenterology. 2018;154:302-18.

28. Peura DA, Riff DS, Snoddy AM, Fennerty MB. Clinical trial: lansoprazole 15 or 30 mg once daily vs. placebo for treatment of frequent nighttime heartburn in self-treating subjects. Aliment Pharmacol Ther. 2009;30:459-68.

29. Fujiwara Y, Habu Y, Ashida K, Kusano M, Higuchi K, Arakawa T. Sleep disturbances and refractory gastroesophageal reflux disease symptoms in patients receiving once-daily proton pump inhibitors and efficacy of twice-daily rabeprazole treatment. Digestion. 2013;88:145-52.

30. Norris V, Baisley K, Dunn K, Warrington S, Morocutti A. Combined analysis of three crossover clinical pharmacology studies of effects of rabeprazole and esomeprazole on 24-h intragastric $\mathrm{pH}$ in healthy volunteers. Aliment Pharmacol Ther. 2007;25:501-10.

31. Fock KM, Teo EK, Ang TL, Chua TS, Ng TM, Tan YL. Rabeprazole vs esomeprazole in non-erosive gastro-esophageal reflux disease: a randomized, double-blind study in urban Asia. World J Gastroenterol. 2005;11:3091-8.

32. Chen MJ, Wu MS, Lin JT, Chang KY, Chiu HM, Liao WC, Chen CC, Lai YP, Wang HP, Lee YC. Gastroesophageal reflux disease and sleep quality in a Chinese population. J Formos Med Assoc. 2009;108:53-60.

33. Vakil N, van Zanten SV, Kahrilas P, Dent J, Jones R. The Montreal definition and classification of gastroesophageal reflux disease: a global evidence-based consensus. Am J Gastroenterol. 2006;101:1900-20. quiz 1943.

34. Kahrilas PJ, Howden CW, Hughes N. Response of regurgitation to proton pump inhibitor therapy in clinical trials of gastroesophageal reflux disease. Am J Gastroenterol. 2011;106:1419-25. quiz 1426.

35. Ryden A, Martin M, Halling K, Niklasson A. Night-time symptoms and their impact on sleep in patients with gastroesophageal reflux disease who have a partial response to proton pump inhibitors: a qualitative patient interview study. Patient. 2013;6:307-14.

\section{Tables}

Table 1 Demographics and baseline characteristics of patients 


\begin{tabular}{lc}
\hline Characteristics & Total (n=750) \\
\hline Age (y) & $51.21(0.51)$ \\
Mean (SD) & $41(18-79 \square$ \\
\hline Median (rang) & $397,52.9 \%$ \\
\hline Male (n, \%) & $24.4(2.9)$ \\
\hline BMI (kg/m ${ }^{2}$ ) & $1.89(0.78)$ \\
\hline Mean (SD) & $1.88(0.78)$ \\
\hline Median (range) & $6.82(0.12)$ \\
\hline Mean nighttime heartburn severity score (SD) & $427(56.9 \%)$ \\
\hline Mean nighttime acid regurgitation severity score (SD) & \\
\hline Mean PSQI score (SD) & \\
PSQI 45 (n, \%) & \\
\hline
\end{tabular}

Data were presented as mean (standard deviation) or $\mathrm{n}(\%)$

BMI, body mass index

PSQI, Pittsburgh Sleep Quality Index

\section{Figures}




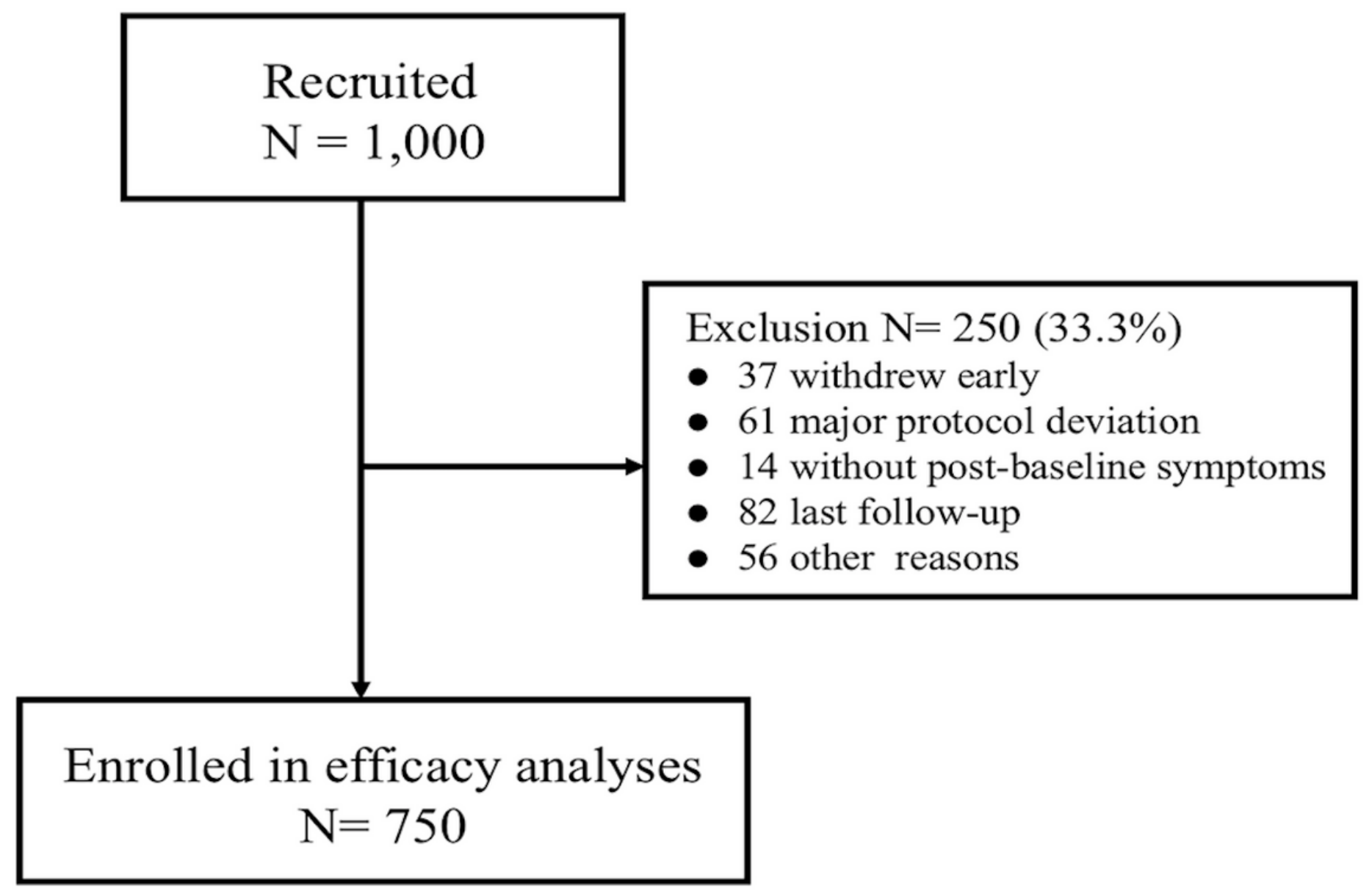

Figure 1

Flow chart of patients.
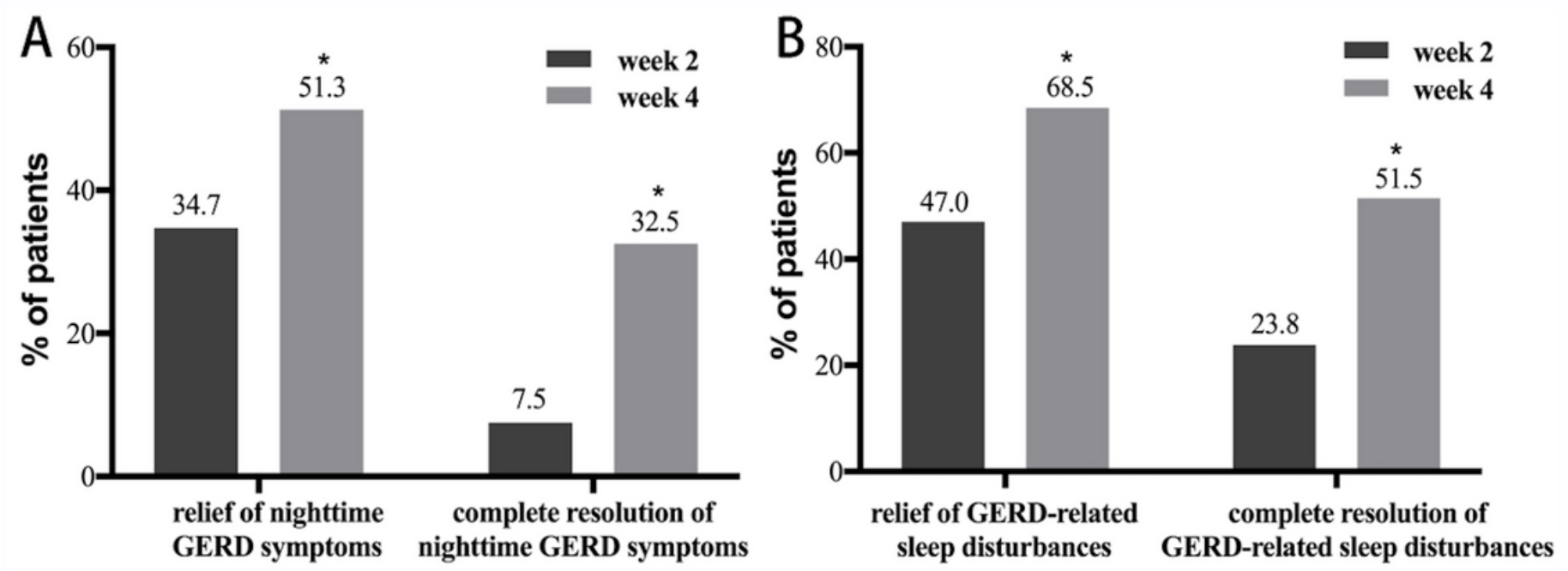

Figure 2 
Effect of PPIs treatment on nighttime GERD symptoms (A) and GERD-related sleep disturbances (B) after 2 and 4 weeks. PPIs treatment over 4 weeks or 2 weeks significantly reduced the frequency of nighttime GERD symptoms, as well as GERD-related sleep disturbances. *P囚.001 versus baseline.
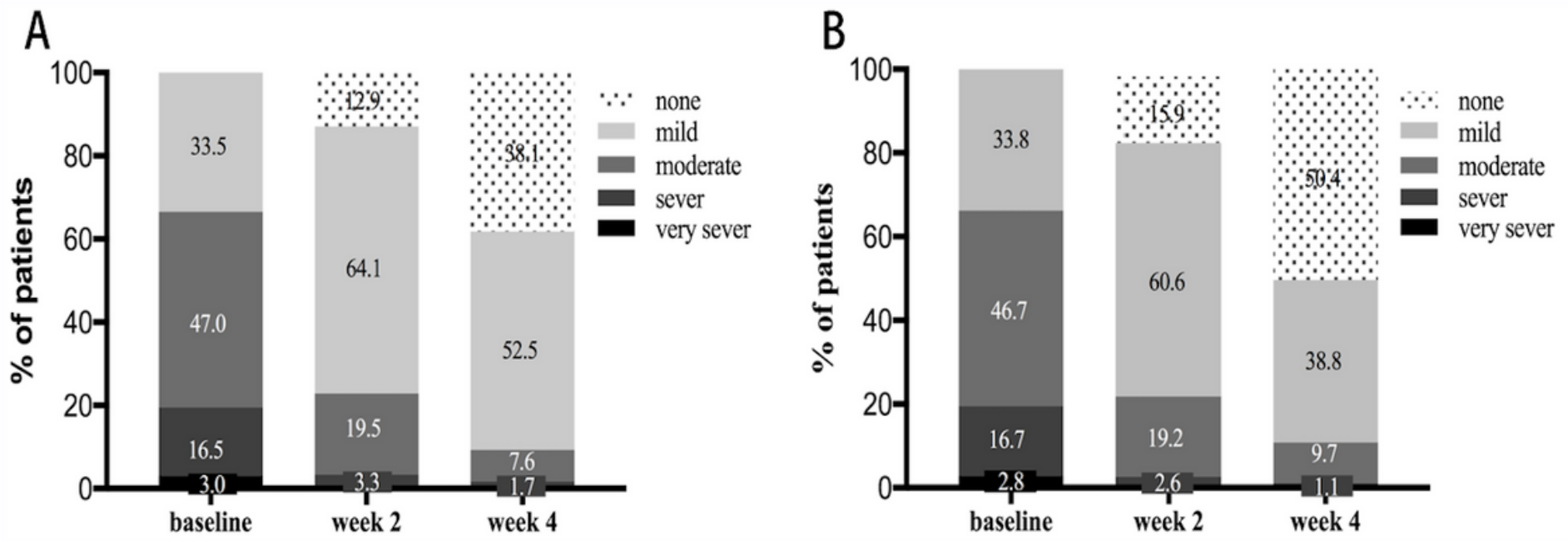

Figure 3

Changes in the distribution of nighttime heartburn severity (A) and nighttime acid regurgitation (B) from baseline to week 4 .
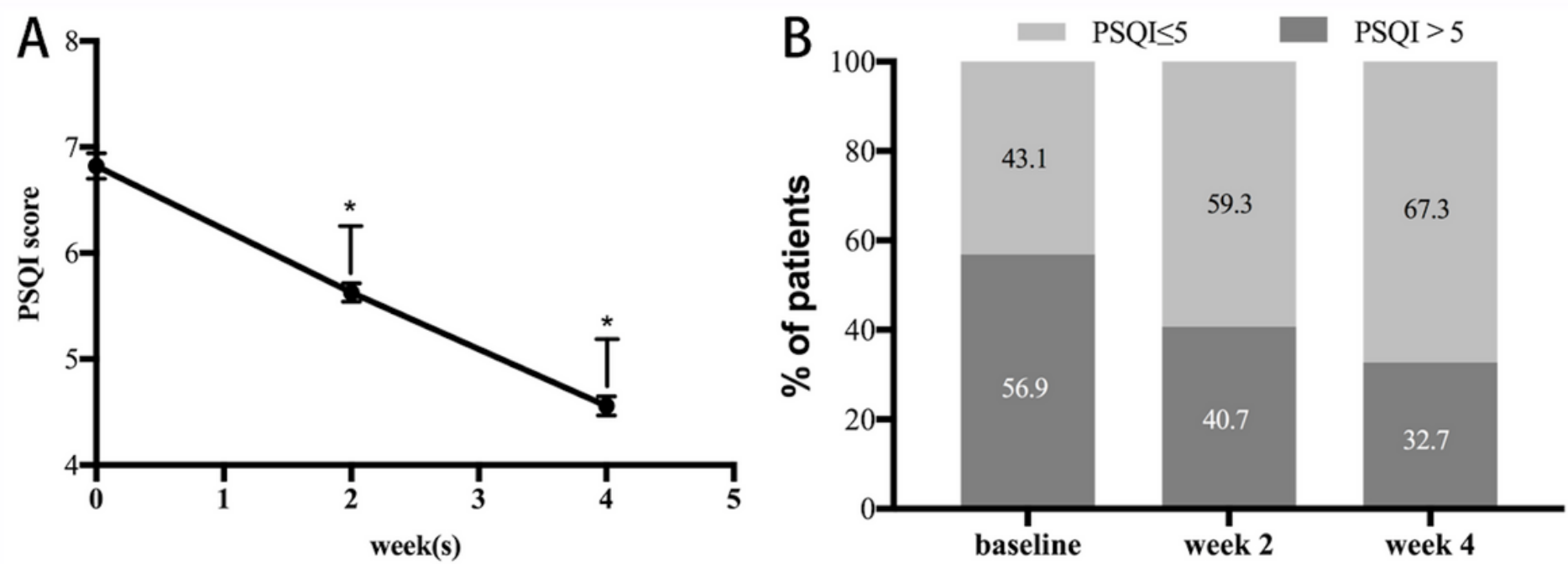

\section{Figure 4}

Changes in the mean PSQI score (A) and proportion of patients with a total PSQI score $₫ 5$ from baseline to week 4 (B). PPIs treatment significantly decreased the mean PSQI score both at week 2 and week 4. PSQI: the Pittsburgh Sleep Quality Index. *P囚.001 versus baseline. 


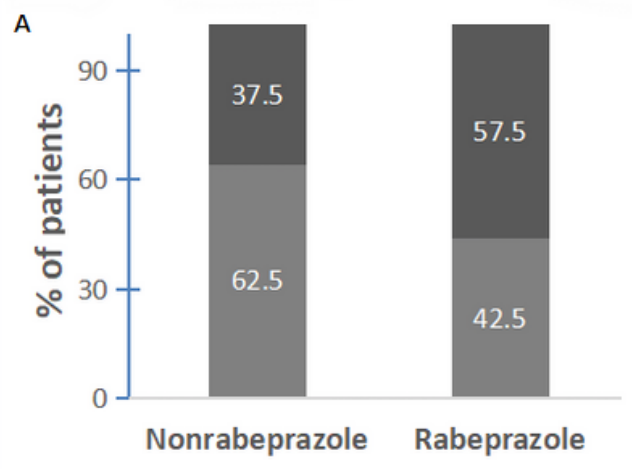

Nighttime GERD symptoms

C

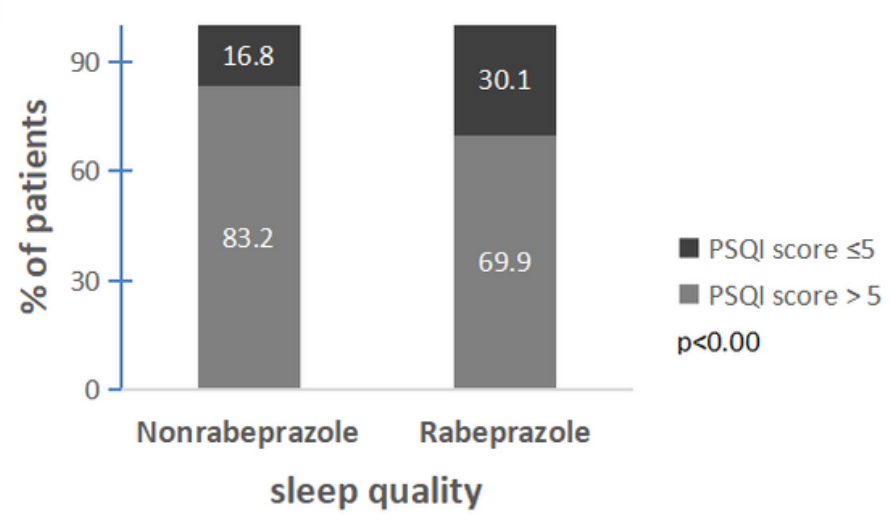

- Respondent

non-respondent $p<0.001$

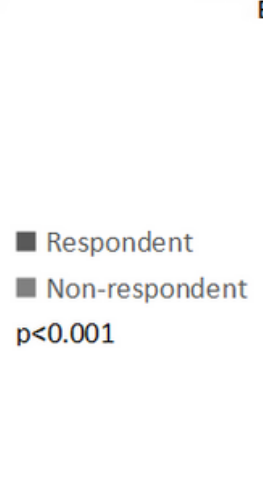

B

- Respondent

Non-respondent $p<0.001$

\section{Figure 5}

Effect of rabeprazole and non-rabeprazole on nighttime GERD symptoms (A), GERD-related sleep disturbances (B) and PSQI score (C) at week 4. 